\title{
Cauchy Problem in the Non-Classical Treatment for One Pseudoparabolic Equation
}

\author{
Ilgar G. Mamedov \\ Institute of Cybernetics, Azerbaijan National Academy of Sciences, Az 1141, Azerbaijan, Baku, st. B. Vahabzade, 9 \\ *Corresponding Author: ilgar-mammadov@rambler.ru
}

Copyright $@ 2014$ Horizon Research publishing all rights reserved.

\begin{abstract}
In the paper, we consider the Cauchy problem for a fifth order pseudoparabolic equation that appears in studying the issues of fluid filtration in fissured media, the moisture transfer in soils and etc. The Cauchy problem with non-classic conditions not requiring the agreement conditions are studied for a discontinuous coefficient pseudoparabolic equation. The equivalence of these conditions with the Cauchy classic condition is substantiated in the case when the solution of the stated problem is sought in S.L.Sobolev anisotropic space $W_{p}^{(3,2)}(G)$.
\end{abstract}

Keywords Cauchy Problem, Pseudoparabolic Equation, Equations With Discontinuous Coefficients

\section{Introduction}

In the present paper, here consider Cauchy problem for one higher order differential equation with discontinuous coefficients. The coefficients in this pseudoparabolic equation are not necessarily differentiable; therefore, there does not exist a formally ad joint differential equation making a certain sense. For this reason, this question cannot be investigated by the well-known methods using classical integration by parts and Riemann functions or classical-type fundamental solutions. Pseudoparabolic equations are attracted for sufficiently adequate description of a great deal of real processes occurring in the nature, engineering and etc. In particular, many processes arising in the theory of fluid filtration in cracked media are described by discontinuous coefficient pseudoparabolic equations. The theme of the present paper, devoted to the investigation Cauchy problem for one differential equations of pseudoparabolic type, according to the above-stated is very actual for the solution of theoretical and practical problems. From this point of view, the paper is devoted to the actual problems of partial differential equations and computational mathematics.

\section{Problem Statement}

Let $G=G_{1} \times G_{2}, \quad G_{k}=\left(0, h_{k}\right), \quad k=\overline{1,2} ; \quad W_{p}^{(3,2)}(G)$, $1 \leq p \leq \infty$ be a space of all functions $u \in L_{p}(G)$ having the generalized derivatives, in S.L.Sobolev`s sense $D_{x}^{i} D_{y}^{j} u \in L_{p}(G), i=\overline{0,3} ; j=\overline{0,2}$, where $D_{t}=\partial / \partial_{t}$ is a generalized differentiation operator in S.L.Sobolev`s sense, $D_{t}^{0}$ is an identical transformation operator. We'll define the norm in S.L.Sobolev anisotropic space $W_{p}^{(3,2)}(G)$ by the following equality

$$
\|u\|_{W_{p}^{(3,2)}(G)}=\sum_{i=0}^{3} \sum_{j=0}^{2}\left\|D_{x}^{i} D_{y}^{j} u\right\|_{L_{p}(G)}
$$

Consider the equation

$$
\begin{aligned}
& \left(V_{3,2} u\right)(x, y) \equiv D_{x}^{3} D_{y}^{2} u(x, y)+a_{2,2}(x, y) D_{x}^{2} D_{y}^{2} u(x, y)+ \\
& +a_{3,1}(x, y) D_{x}^{3} D_{y} u(x, y)+a_{2,1}(x, y) D_{x}^{2} D_{y} u(x, y)+ \\
& +a_{1,2}(x, y) D_{x} D_{y}^{2} u(x, y)+a_{3,0}(x, y) D_{x}^{3} u(x, y)+ \\
& +\sum_{\substack{i=0 \\
i+j<3}}^{2} \sum_{\substack{j=0 \\
i, j}} a_{i, y}(x, y) D_{x}^{i} D_{y}^{j} u(x, y)=Z_{3,2}(x, y),(x, y) \in G,
\end{aligned}
$$

where $u(x, y)$ is a desired function from $W_{p}^{(3,2)}(G)$.

Some classes of boundary value problems for equation (1) in definite sense are stated similar to the known boundary value problems for the parabolic equation $D_{y} u(x, y)=D_{x}^{2} u(x, y)$. Therefore, many authors call the equation of the form (1) a pseudoparabolic equation. Notice that the equation under consideration is a generalization of many model equations of some processes (for example, of heat conductivity equation, string vibration equation, telegraph equation, generalized moisture transfer equation, Boussenesq-Love equation and etc.).

In particular, many processes arising in theory of fluid filtration in fissured media [1-2] are described by pseudoparabolic equations with discontinuous coefficients [3-4]. Such equations arise while describing a lot of real processes occurring in nature and engineering [5-9]. Similar situations hold by studying the phenomena that happen in plasma, in the processes of heat propagation, moisture 
transfer in soils, the fluid filtration in porous - fissured media and also in the problems of mathematical biology and demography [10].

For equation (1) state the Cauchy problem in the form:

$$
\begin{gathered}
\left(V_{3, j} u\right)(x) \equiv D_{x}^{3} D_{y}^{j} u(x, S(x))=Z_{3, j}(x), x \in G_{1}, \\
\left(V_{i, 2} u\right)(y) \equiv D_{x}^{i} D_{y}^{2} u(v(y), y)=Z_{i, 2}(y), y \in G_{2}, \\
V_{i, j} u \equiv D_{x}^{i} D_{y}^{j} u\left(0, h_{2}\right)=Z_{i, j}, i=\overline{0,2} ; j=\overline{0,1},
\end{gathered}
$$

where $S: \bar{G}_{1} \rightarrow \bar{G}_{2}$ is an absolutely continuous, strictly decreasing function. $S(0)=h_{2}, S\left(h_{1}\right)=0, v: \overline{G_{2}} \rightarrow \overline{G_{1}}$ is a function inverse to the function $y=S(x)$, and the derivative $S_{x}(x)$ is bounded on $G_{1}$ (see. Figure 1):

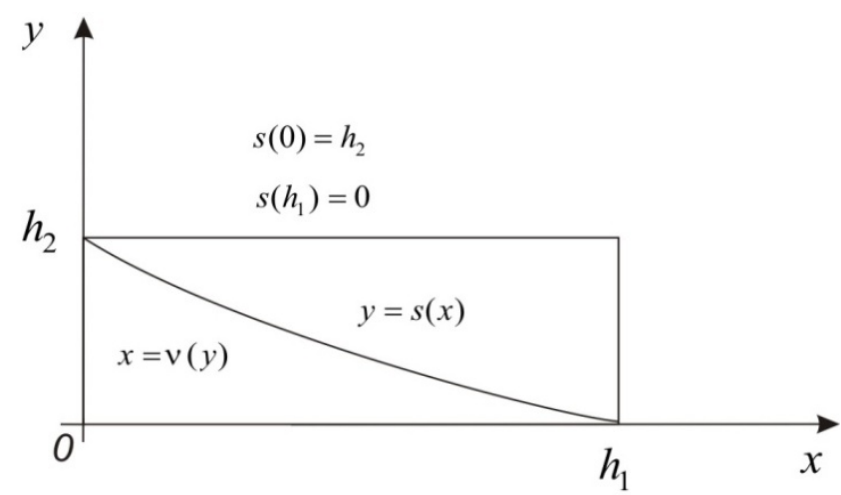

Figure 1. Graphic representation of the function $S: \bar{G}_{1} \rightarrow \bar{G}_{2}$ and its inverse function $v: \overline{G_{2}} \rightarrow \overline{G_{1}}$.

Let the conditions $a_{i, j}(x, y) \in L_{p}(G), i=\overline{0,2} ; \quad j=\overline{0,1}$ be fulfilled and there exist the functions

$$
\begin{aligned}
& a_{i, 2}^{0}(x) \in L_{p}\left(G_{1}\right), i=\overline{0,2}, \\
& a_{3, j}^{0}(y) \in L_{p}\left(G_{2}\right), j=\overline{0,1}
\end{aligned}
$$

such that $\left|a_{i, 2}(x, y)\right| \leq a_{i, 2}^{0}(x),\left|a_{3, j}(x, y)\right| \leq a_{3, j}^{0}(y)$ almost everywhere on $G$;

$$
\begin{gathered}
Z=\left(Z_{3,2}, Z_{3,0}, Z_{3,1}, Z_{0,2}, Z_{1,2}, Z_{2,2}, Z_{0,0}, Z_{1,0}, Z_{2,0}, Z_{0,1}, Z_{1,1}, Z_{2,1}\right) \in \\
\in E_{p}^{(3,2)} \equiv L_{p}(G) \times L_{p}\left(G_{1}\right) \times L_{p}\left(G_{1}\right) \times \\
\times L_{p}\left(G_{2}\right) \times L_{p}\left(G_{2}\right) \times L_{p}\left(G_{2}\right) \times R \times R \times R \times R \times R \times R,
\end{gathered}
$$

where $R$ is a space of real numbers.

Up to now, in all the papers known in references the Cauchy problem for equation (1) was stated and studied in the classic form:

$$
\left\{\begin{array}{l}
D_{x}^{i} D_{y}^{j} u(x, S(x))=Z^{(i, j)}(x), x \in G_{1}, i=\overline{0,2}, \quad j=\overline{0,1}, \\
D_{x}^{2} D_{y}^{2} u(v(y), y)=Z^{(4)}(y), \quad y \in G_{2} .
\end{array}\right.
$$

\section{Methodology}

There with, the important principal moment is that the considered equation possesses discontinuous coefficients satisfying only some $p$-integrability and boundedpess conditions i.e. the considered pseudoparabolic operator 3,2 has no traditional conjugated operator. In other words, the Riemann function for this equation can't be investigated by the classical method of characteristics. In the papers [11-12] the Riemann function is determined as the solution of an integral equation. This is more natural than the classical way for deriving the Riemann function. The matter is that in the classic variant, for determining the Riemann function, the rigid smooth conditions on the coefficients of the equation are required.

The Riemann's method does not work for differential equations with discontinuous coefficients.

In the present paper, a method that essentially uses modern methods of the theory of functions and functional analysis is worked out for investigations of such problems. In the main, this method it requested in conformity to pseudoparabolic equations of fifth order. Notice that, in this paper the considered equation is a generation of many model equations of some processes (for example, heat-conductivity equations , telegraph equation, Aller's equation, moisture transfer generalized equation, Manjeron equation, equation, string vibrations equations and etc).

Show that conditions (2) and (3) are equivalent. Indeed let $u \in W_{p}^{(3,2)}(G)$ be a solution of problem (1), (2). Show that it is a solution of problem (1), (3) for some $Z^{(i, j)}(x), i=\overline{0,2}, \quad j=\overline{0,1}, Z^{(4)}(y)$. For that consider the identities:

$$
\begin{gathered}
u(x, S(x))=u\left(0, h_{2}\right)+\int_{0}^{x} D_{x} u(\tau, S(\tau)) d \tau+\int_{h_{2}}^{S(x)} D_{y} u(v(\xi), \xi) d \xi, \\
D_{x} u(x, S(x))=D_{x} U\left(0, h_{2}\right)+\int_{0}^{x} D_{x}^{2} u(\tau, S(\tau)) d \tau+\int_{h_{2}}^{S(x)} D_{x} D_{y} u(v(\xi), \xi) d \xi, \\
D_{y} u(x, S(x))=D_{y} u\left(0, h_{2}\right)+\int_{0}^{x} D_{x} D_{y} u(\tau, S(\tau)) d \tau+\int_{h_{2}}^{S(x)} D_{y}^{2} u(v(\xi), \xi) d \xi,
\end{gathered}
$$




$$
\begin{gathered}
D_{x} D_{y} u(x, S(x))=D_{x} D_{y} u\left(0, h_{2}\right)+\int_{0}^{x} D_{x}^{2} D_{y} u(\tau, S(\tau)) d \tau+\int_{h_{2}}^{S(x)} D_{x} D_{y}^{2} u(v(\xi), \xi) d \xi, \\
D_{x}^{2} u(x, S(x))=D_{x}^{2} u\left(0, h_{2}\right)+\int_{0}^{x} D_{x}^{3} u(\tau, S(\tau)) d \tau+\int_{h_{2}}^{S(x)} D_{x}^{2} D_{y} u(v(\xi), \xi) d \xi, \\
D_{x}^{2} D_{y} u(x, S(x))=D_{x}^{2} D_{y} u\left(0, h_{2}\right)+\int_{0}^{x} D_{x}^{3} D_{y} u(\tau, S(\tau)) d \tau+\int_{h_{2}}^{S(x)} D_{x}^{2} D_{y}^{2} u(v(\xi), \xi) d \xi,
\end{gathered}
$$

As $u \in W_{p}^{(3,2)}(G)$ is the solution of problem (1), (2), then from (4)-(9) it follows that

$$
\begin{gathered}
D_{x}^{2} D_{y} u(x, S(x))=Z_{2,1}+\int_{0}^{x} Z_{3,1}(\tau) d \tau+\int_{h_{2}}^{S(x)} Z_{2,2}(\xi) d \xi=\breve{Z}^{2,1}(x), \\
D_{x}^{2} u(x, S(x))=Z_{2,0}+\int_{0}^{x} Z_{3,0}(\tau) d \tau+\int_{h_{2}}^{S(x)} \breve{Z}^{(2,1)}(v(\xi)) d \xi=\breve{Z}^{2,0}(x), \\
D_{x} D_{y} u(x, S(x))=Z_{1,1}+\int_{0}^{x} \breve{Z}^{(2,1)}(\tau) d \tau+\int_{h_{2}}^{S(x)} Z_{1,2}(\xi) d \xi=\breve{Z}^{(1,1)}(x), \\
D_{y} u(x, S(x))=Z_{0,1}+\int_{0}^{x} \breve{Z}^{(1,1)}(\tau) d \tau+\int_{h_{2}}^{S(x)} Z_{0,2}(\xi) d \xi=\breve{Z}^{(0,1)}(x), \\
D_{x} u(x, S(x))=Z_{1,0}+\int_{0}^{x} \breve{Z}^{(2,0)}(\tau) d \tau+\int_{h_{2}}^{S(x)} \breve{Z}^{(1,1)}(v(\xi)) d \xi=\breve{Z}^{(1,0)}(x), \\
u(x, S(x))=Z_{0,0}+\int_{0}^{x} \breve{Z}^{(1,0)}(\tau) d \tau+\int_{h_{2}}^{S(x)} \breve{Z}^{(0,1)}(v(\xi)) d \xi=\breve{Z}^{(0,0)}(x)
\end{gathered}
$$

This means that $u(x, y)$ is the solution of problem (1), (3) for

$$
\begin{aligned}
Z^{(0,0)}(x)= & \breve{Z}^{(0,0)}(x), Z^{(1,0)}(x)=\breve{Z}^{(1,0)}(x) Z^{(0,1)}(x)=\breve{Z}^{(0,1)}(x), Z^{(1,1)}(x)=\breve{Z}^{(1,1)}(x), \\
& Z^{(2,0)}(x)=\breve{Z}^{(2,0)}(x), Z^{(2,1)}(x)=\breve{Z}^{(2,1)}(x), Z^{(4)}(y)=Z_{2,2}(y) .
\end{aligned}
$$

Vice - versa, if $u \in W_{p}^{(3,2)}(G)$ is the solution of problem (1), (3), then from the identities (4)-(9) we have

$$
\begin{gathered}
Z^{(2,1)}(x)=Z^{(2,1)}(0)+\int_{0}^{x} D_{x}^{3} D_{y} u(\tau, s(\tau)) d \tau+\int_{h_{2}}^{S(x)} Z^{(4)}(\xi) d \xi, \\
Z^{(2,0)}(x)=Z^{(2,0)}(0)+\int_{0}^{x} D_{x}^{3} u(\tau, s(\tau)) d \tau+\int_{h_{2}}^{S(x)} Z^{(2,1)}(v(\xi)) d \xi, \\
Z^{(1,1)}(v(y))=Z^{(1,1)}(0)+\int_{0}^{v(y)} Z^{(2,1)}(\tau) d \tau+\int_{h_{2}}^{S(x)} D_{x} D_{y}^{2} u(v(\xi), \xi) d \xi, \\
Z^{(0,1)}(v(y))=Z^{(0,1)}(0)+\int_{0}^{v(y)} Z^{(1,1)}(\tau) d \tau+\int_{h_{2}}^{y} D_{y}^{2} u(v(\xi), \xi) d \xi,
\end{gathered}
$$




$$
Z^{(1,0)}(x)=Z^{(1,0)}(0)+\int_{0}^{x} Z^{(2,0)}(\tau) d \tau+\int_{h_{2}}^{S(x)} Z^{(1,1)}(v(\xi)) d \xi, \quad Z^{(0,0)}(x)=Z^{(0,0)}(0)+\int_{0}^{x} Z^{(1,0)}(\tau) d \tau+\int_{h_{2}}^{S(x)} Z^{(0,1)}(v(\xi)) d \xi
$$

Hence we find that $u(x, y)$ is the solution of problem (1), (2) for

$$
\begin{gathered}
Z_{0,0}=Z^{(0,0)}(0), Z_{1,0}=Z^{(1,0)}(0), Z_{0,1}=Z^{(0,1)}(0), \\
Z_{1,1}=Z^{(1,1)}(0), Z_{2,0}=Z^{(2,0)}(0), Z_{2,1}=Z^{(2,1)}(0), Z_{2,2}(y)=Z^{(4)}(y), Z_{3,0}(x)=\frac{d}{d x} F^{(1)}(x), \\
F^{(1)}(x)=Z^{(2,0)}(x)-\int_{h_{2}}^{S(x)} Z^{(2,1)}(v(\xi)) d \xi \\
Z_{3,1}(x)=\frac{d}{d x} F^{(2)}(x), F^{(2)}(x)=Z^{(2,1)}(x)-\int_{h_{2}}^{S(x)} Z^{(4)}(\xi) d \xi, \\
Z_{0,2}(y)=\frac{d}{d y} F^{(3)}(y), F^{(3)}(y)=Z^{(0,1)}(v(y))-\int_{0}^{v(y)} Z^{(1,1)}(\tau) d \tau \\
Z_{1,2}(y)=\frac{d}{d y} F^{(4)}(y), F^{(4)}(y)=Z^{(1,1)}(v(y))-\int_{0}^{v(y)} Z^{(2,1)}(\tau) d \tau
\end{gathered}
$$

Thus, conditions (2) are equivalent to the classic form Cauchy conditions (3). Therefore, the classic form Cauchy problems (1), (3) and in non-classical treatment (1), (2) are equivalent in the general case. However, the Cauchy problem in non-classical treatment (1), (3) is more natural by statement than problem (1), (3). In the non-classic treatment no additional conditions of "agreement" type are imposed on the right sides

$$
\begin{gathered}
Z_{3,2}(x, y) \in L_{p}(G), Z_{3, j}(x) \in L_{p}\left(G_{1}\right), Z_{i, 2}(y) \in L_{p}\left(G_{2}\right), \\
Z_{i, j} \in R, i=\overline{0,2} ; j=\overline{0,1}
\end{gathered}
$$

this enables to expect that the operator of problem (1), (2) gives homeomorphism. However, in case (1), (3) we can't expect it. The matter is that in this case, for the existence of the solution it is necessary that the conditions

$$
F^{(1)}(x) \in W_{p}^{(1)}\left(G_{1}\right), F^{(2)}(x) \in W_{p}^{(1)}\left(G_{1}\right), F^{(3)}(y) \in W_{p}^{(1)}\left(G_{2}\right)
$$

and $F^{(4)}(y) \in W_{p}^{(1)}\left(G_{2}\right)$, that may be considered as "agreement" conditions connecting the given functions

$$
Z^{(0,1)}(x), Z^{(1,1)}(x), Z^{(2,0)}(x), Z^{(2,1)}(x), Z^{(4)}(y)
$$

be fulfilled.

\section{Result}

So, the classic form Cauchy problem (1), (3) and in non-classical treatment (1), (2) are equivalent in the general case. However, the Cauchy problem in non-classical treatment (1), (2) is more natural by the statement than problem (1), (3). This is connected with the fact that in the statement of problem (1), (2) the right sides of Cauchy conditions don't require additional conditions of agreement type. Note that such boundary value problems in non-classical treatment were considered in the author's papers [13-17]. 


\section{Discussion and Conclusions}

In the paper a non-classical type Cauchy problem is substantiated for a pseudoparabolic equation with non-smooth coefficients and with a fifth order dominating derivative. Classic Cauchy conditions are reduced to non-classic Cauchy conditions by means of integral representations. Such statement of the Cauchy problem has several advantages:

1) No additional agreement conditions are required in this statement;

2) One can consider this statement as a Cauchy problem formulated in the S.L. Sobolev anisotropic space $W_{p}^{(3,2)}(G)$;

3) In this statement the considered equation is a generalization of many model equations of some processes (e.g. telegraph equation, string vibrations equations, heat-conductivity equations , Aller's equation, moisture transfer generalized equation, Manjeron equation, Boussinesq-Love equation and etc.).

\section{REFERENCES}

[1] G.I.Barenblatt, Yu.P.Zheltov, I.N.Kochina, "On main representations of theory of homogeneous fluids filtration in fissued rocks", Prikl. mat. i mekh. 1960, vol. 25. issul 5, pp.852-864

[2] V.D.Gilev, G.A.Shadrin, "Construction of fundamental solution for an equation describing fluids motion in fissued media", Vichisliteluaya matem. i programmirovaniye, V.I.Lenin MSPI publishe. 1976, issue 4. pp.102-111.

[3] M.Kh.Shkanukov, "On some boundary value problems for a third order equation arizing by modeling fluid filtration in porous media", Differen. Uravn. 1982, vol.18, No 4, pp.689-699.

[4] M.Kh.Shkanukov, "On some boundary value problems for a third order equation and extremal properties of its solutions", Differen. Uravn. 1983, vol.19, No 1, pp.145-152.

[5] A.P.Soldatov, M.Kh.Shkhanukov, "Boundary value problems with A.A.Samarsky general nonlocal condition for higher order pseudoparabolic equations", Dokl. AN USSR, 1987, vol.297, No 3. pp.547-552 .

[6] W.Rundell, "The uniqueness class for the Cauchy problem for pseudoparabolic equations", Proc. Amer. Match. Soc., 1979, vol.76, No 2, pp. 253-257.

[7] D.Colton, "Pseudoparabolic equations in one space variable", J. Different. Equat. 1972, vol.12, No 3, pp.559-565.

[8] G.V.Demidenko, "The Cauchy problem for pseudoparabolic systems", Sib. Matem. Zhurnal. 1997, vol.38, No 6, pp.1251-1266.

[9] A.L.Gladkov, "Uniquenenes of the Cauchy problem solution for some quasilinear pseudoparabolic equations", Matem. Zametki. 1996, vol.60, No 3, pp.356-362.

[10] A.M.Nakhushev, Equations of mathematical biology M.: Visshaya shkola 1995, $304 \mathrm{p}$.

[11] S.S.Akhiev, "Fundamental solution to some local and non local boundary value problems and their representations ", Dokl. AN USSR, 1983, vol.271, No 2, pp.265-269.

[12] V.I.Zhegalov, E.A.Utkina, "On a third order pseudoparabolic equation”, Izv. Vuzov, Matem., 1999, No10, pp.73-76.

[13] I.G.Mamedov, "One Goursat problem in a Sobolev space", Izv. Vuzov. Matem. 2011, No 2, pp.54-64.

[14] I.G.Mamedov, "Goursat problem in the non-classical treatment for a sixth order pseudoparabolic equation", Universal Journal of Computational Mathematics,2013, vol.1, No 1, pp.15-18.

[15] I.G.Mamedov, "A fundamental solution to the Cauchy problem for a fourth- order pseudoparabolic equation",Computational Mathematics and Mathematical Physics, 2009, volume 49, Issue 1, pp 93-104.

[16] I.G.Mamedov, "A mixed problem with Bitsadze - Samarsky and Samarsky-Ionkin type conditions, arising by modeling fluid filtration in fussued media", Izv. NAS of Azerb. Ser. Fiz-tekhn. i matem. nauk., 2006, vol. XXVI, , No 3, pp. 32-37.

[17] I.G.Mamedov, "Investigation of a problem with integro-multipoint boundary conditions for generalized moisture transfer equation", Izv. NAS of Azerb. Ser. Fiz-tekhn. i matem. nauk., 2007, vol. XXVII, No 3, pp. 121-126. 